\title{
A Practice Based Chronic Pain Management Registry (CPMR): Structure and Content of Proposed Patient and Patient/Provider Platforms
}

Paul C Langley, PhD

Adjunct Professor, College of Pharmacy, University of Minnesota

\begin{abstract}
Previous commentaries in the Formulary Evaluation section of INNOVATIONS in Pharmacy have pointed to the difficulties of establishing the credibility of trial-based and modeled claims for therapy interventions. Claims for interventions in the management of chronic pain are no exception. To meet this challenge, the Chronic Pain Management Registry (CPMR) has been designed to provide an evidence base for physician practices and health care decision makers to evaluate the impact of pain management interventions. The purpose of this commentary is to detail the development, structure and content of the CPMR in two versions: (i) a patient response version and (ii) a combined patient/provider response version. The CPMR has a potentially critical role to play in providing a framework for the effective auditing of practice compliance in the prescribing and monitoring of opioids in the management of chronic pain. The CPMR tracks, with on-line input from the patient and the treating physician, the process and outcomes of therapy interventions. These reports cover the overall pain experience of patients as well as pain intensity and functional status by eleven specific pain locations, covering both pharmacological and non-pharmacological interventions. Prior to each practice visit patients complete reports which are entered to the CPMR with a summary transmitted to the physician practice. Over time, these reports track the cumulative response to therapy as well as the perception of the patients as to whether or not the therapy has led to any substantive improvement in activity limitations, symptoms and quality of life. A particular focus of the CPMR is on monitoring and evaluating the experience with, and impact of opioid medications, to include the effectiveness of opioid formulations on reducing pain intensity and improving functional status, including an intensive assessment of the potential for and experience of opioid substance abuse for individual patients. The CPMR can also support monthly reports to the practice to summarize patient throughput, the response to care by target pain patients and profiles of opioid use and abuse. The CPMR can be customized to meet the needs of individual practices.
\end{abstract}

Keywords: Chronic Pain Management Registry, CPMR, claims credibility, opioid evaluation, medication abuse, pain management

\section{Introduction}

The choice of therapy option and associated outcomes for patients experiencing chronic pain has received increasing attention over the past decade, with a major focus of public policy on the blanket use, potential abuse and the actual clinical efficacy of opioid medications. At the same time, there has been increased attention given to the quality of care and outcomes of health care interventions in chronic pain and a range of other disease states. This is seen in the emphasis on how value in health care is paid for and coordinated with the shift toward increased collaboration, outcome-based payments and new benefit designs ${ }^{1}$. As an example under the Patient Protection and Affordable Care Act, the Centers for Medicare and Medicaid Services (CMS) have initiated a number of programs and underwritten a range of quality metrics to meet the three aims of better care, smarter spending, and healthier people. In the Medicare population, for example, the Merit Based Incentive Payment System

Corresponding author: Paul C Langley, PhD

Adjunct Professor

College of Pharmacy, University of Minnesota

Director, Maimon Research LLC

5061 North Apache Hills Trail, Tucson, AZ 85750

Email: langley@maimonresearch.com
(MIPS) offers substantial financial incentives for healthcare providers to report: (i) a range of quality metrics, (ii) activities to support comprehensive healthcare information and (iii) care improvement activities. There has also been a major expansion in the number of reportable and billable Current Procedure Terminology (CPT) codes (e.g., CPT 96127 Brief Emotional/Behavioral Assessment) designed to support more focused patient assessment and to facilitate outcomes tracking. Appropriately used, these promise a financial return to physician practices to meet the increased demands for greater efficiency in resource allocation and measured greater effectiveness in the provision of health care.

The CPMR is available in two versions: (i) a patient only response platform and (ii) a patient and provider response platform. These options recognize that pain practices may not have the resources or may not be willing in the first instance to commit to a registry where data are captured from both patients and physician practices.

Both versions of the CPMR generate summary reports on individual patients to support ongoing clinical assessments of response to therapy as well as customized practice management reports for target patient groups. At the same time the adoption of a CPMR by a specialist pain practice or primary care group provides an interface to a proposed companion medical marijuana dispensary registry. This links 
provider certification of a patient for medical marijuana use to outcomes reported by patients who have enrolled in a medical marijuana dispensary. Credible evidence on outcomes from a cannabis dispensary registry is important as it has the potential to provide justification for switching patients from opioids in the medium and long-term management of chronic pain. It is worth emphasizing that severe pain is the single most important condition reported to dispensaries, accounting for $75-85 \%$ of clients.

\section{From Process to Outcome}

A critical element in practice-based performance, envisaged in the increased focus by agencies such as CMS in switching from process to outcome measures, is to track patients over the course of their treatment. It is becoming incumbent upon physician practices and health care systems not only to track patients over the course of their disease but to ensure that, wherever possible, tracking metrics utilize validated patient reported outcomes (PRO) instruments in their management and evaluation of the ongoing patient response to therapy.

The increased emphasis on tracking patients reflects a longstanding concern with the evidentiary standards for clinical decisions in chronic pain treatment practice. This concern reflects, not only the limited evidence for the medium and longterm impact of treatment interventions but the credibility of the published evidence. The latest CDC Guidelines for Prescribing Opioids for Chronic Pain point out that evidence on long-term opioid therapy for chronic pain outside of end-of-life care remains limited, with insufficient evidence to determine long-term benefits versus no opioid therapy ${ }^{2}$. Although clinical trial data would suggest that risk for serious harms appear to be dose dependent, the evidence is typically short-term with most trials only reporting outcomes for 12 weeks or less. At best, the evidence would suggest that opioids are only moderately effective for pain relief and with small benefits for functional outcomes. This criticism applies with equal force to established opioid formulations as well as to the abuse deterrent opioid formulations that are entering the market place. Formulary committees cannot judge the competing merits claimed for abuse deterrent formulations in the absence of credible evidence, both from randomized clinical trials (RCTs) as well as practice based observational studies.

In the absence of adequate evidence, the risk is that a high percentage of patients will discontinue opioid use because of lack of efficacy and adverse events. If this is the case then the question might be raised as to the advisability of introducing patients to these medications in the first place or at least managing these patients more intensively. At the same time, blanket promotion and uninformed use of opioids and other controlled substances in chronic pain, all too often endorsed by manufacturers, puts patients at avoidable (in retrospect) risk of abuse, dependency and utilization of more high risk illegal substances. While botanical cannabis provided through a dispensary may offer a viable option to opioids as a mainstay of chronic pain management, what is missing is the evidence base for botanical cannabis to support physician decision making.

Concerns with the limited evidence for medium and long-term outcomes in chronic pain are not restricted to pharmacological interventions. A similar dearth of evidence applies to a number of non-pharmacological interventions. In the case of radiofrequency ablation, for example, the most recent systematic review for sacroiliac joint interventions pointed to the variable quality of the evidence, including inconsistencies in diagnostic accuracy, small sample sizes to support study claims and a lack of claims replication. Similar concerns apply to the evidence for cooled radiofrequency ablations, programmable pumps, percutaneous vertebroplasty and kyphoplasty ${ }^{3} 45678$ . Even where single studies claim medium and even long-term benefits, the focus is typically on pain relief with little attention (if any) directed to the functional status of patients by pain location.

A further concern, and this applies to both pharmacological and non-pharmacological pain interventions, is heterogeneity in response to pain interventions and how this response varies over time. If, for example, nerve ablation is seen as initially preferable to drug therapy, the question still remains as to whether patients who achieve only a partial response require supplementary pharmacological treatment to reduce pain and achieve functional improvement. At the same time, over the medium to long-term the option may be between further nerve ablation or switching to a pharmacological therapy including botanical cannabis.

Unfortunately, few physician practices are equipped to monitor and report patient outcomes, as well as reporting at a practice level on the response to therapy by target patient groups. Electronic medical records (EMRs) are not designed to report on cumulative response to therapy.. More to the point: EMRs are deficient in their inability to provide and interpret patient reported outcomes (PROs) from validated instruments and to track these over time in terms of a clinical impact. This is of importance in the treatment of chronic pain where the clinical evidence for long term benefit of competing interventions is limited in terms of both pain intensity and functional status by pain location.

At the same time, few pain practices are equipped to meet recommendations for effective opioid prescribing, to justify the introduction of opioids as a therapy option and to support the ongoing medium and long-term utilization of opioids. While there are guideline recommendations in place to support the prescription of opioids in the more effective treatment of noncancer pain, their application appears limited. A major impetus in the development of the CPMR has been to provide a framework for the more effective management of chronic pain and the prescribing of opioids and opioid substitutes. Concerns with physician and practice compliance were at the forefront in platform design with the role of the platform, particularly given 
concerns with potential legal liability, of providing a robust audit trial to track the interactions between the patient and the treating physician. The recommendations of the American Society of Interventional Pain Physicians (ASIPP) were of particular assistance ${ }^{9}$.

\section{Customization}

Apart from the option of a patient as opposed to a providerpatient version of the CPMR, the registry structure retains the flexibility for customization to meet the needs of pain practices. Additional items can be included (e.g., specific comorbidities, specific line items) with on-line screen modifications, to include modifications to the registry data dictionary. If elements of the registry are felt to involve an undue burden on patients and/or providers as respondents, these can also be modified without putting the basic integrity of the registry data collection and reporting at risk.

\section{Patient Compliance and Opioid Prescribing}

Establishing an audit trail as part of a chronic pain management platform allows both the physician practice and other interested parties to assess therapy decisions in providing an evidence base that physicians can point to in support of those decisions. The proposed registry is ideally suited to creating audit trails. Given standards in place for tracking and monitoring the prescribing of opioids, the CPMR is ideally suited to support third party audits of physician practice. The CPMR provides a baseline profile of the patient's pain status, prior experience of opioids, attitudes to medications and the likelihood of aberrant medication behavior to support a decision to introduce and monitor opioid use. The CPMR tracks the response to therapy, in terms of both clinical response and the patent's belief in the efficacy of the therapy choice, together with the patient reporting aberrant medication behaviors.

At the same time the patient and physician practice version documents therapy changes, adverse events together with reasons for tapering and/or discontinuation of therapy. The CPMR documents whether or not alternative to opioid medication have been considered and the reasons for a patient not scheduling further visits.

\section{Credibility of Medical Marijuana}

The evidence base for the impact of medical marijuana, prescription marijuana products as well as botanical cannabis, is both fragmented and incomplete for the range of conditions that are typically treated under patient certification. Previous commentaries have pointed to the need for a more credible base, proposing that dispensaries establish registries to track patients ${ }^{10} 11 \mathrm{12}$. If we accept that dispensaries have a duty of care to their patients, then establishing a registry should establish links to the providers with feedback on response to the choice of cannabis formulation and the delivery regimen. Although guidelines are only in their early stages, the consensus view appears to be that patients should start with a high CBD (cannabidiol) and low THC (tetrahydrocannabinol) ratio and titrate to an effective dose. Ideally, it is argued, THC should be avoided altogether due to is psychoactive properties with patients only on a CBD regimen, which may include other potentially beneficial non-THC related cannabinoids. Whether these recommendations are sustainable is an open question.

With the passing of the 2018 Farm Bill and the legalization of hemp the door has opened (at least partially) for the production of hemp-based products and extending hemp research by including it under the Critical Agricultural Materials Act. This does not mean that hemp-based CBD is legalized although it has been removed from its schedule 1 status under the Controlled Substances Act. The legislation creates exceptions to its status. Any cannabinoid derived from hemp, including vaporizing CBD oils for potential pain relief, must be produced in accordance with the legislative requirements, including state regulations. There are, in fact, hemp-based cannabinoid products already produced in certain states for consumption within that state.

The (constrained) legalization of hemp-CBD and the potential for a more widespread utilization of derivative products such as hemp CBD oil, means that physicians will have access to a range of products that are potentially beneficial in the treatment of severe and chronic pain. The stumbling block is still, however, the evidence base. Hence the case for a chronic pain registry to provide the framework for tracking the use of hemp-derived cannabinoid products. At present, under medical marijuana legislation these would be provided through a licensed dispensary. This opens the possibility of the registry linking to a provider to support the auditing of therapy response.

Unfortunately, until new compounds are approved that allow us to put opioids to one side physicians are faced with the decision to balance their risks and benefits; a decision which is made all the more difficult by the absence of evidence on the long-term impact of opioids and cannabinoids on both pain levels and functional status. At the same time, physicians have to be aware that blanket denials for opioid utilization are becoming more common. The CPMR attempts to provide a middle ground. As well as providing an evidence base that physicians may utilize to defend opioid and cannabinoid utilization decisions and meet compliance standards, the CPMR provides feedback to the physician to monitor outcomes, including those from cannabinoids. The CPMR, therefore, not only provides evidence to support an initial prescribing decision based on medical necessity but continues to provide evidence to support (or deny) continued opioid prescribing. Access to documentation to support prescribing can also support appeals where opioid prescribing has been denied. This provides additional flexibility in challenging third-party decisions when the treating physician has determined and can justify a particular therapy choice on grounds of medical necessity. 


\section{The Evidence Base}

There are a number of features of chronic pain populations that support the need for a registry. A recent study by Romanelli et al illustrates the heterogeneous characteristics of chronic pain populations ${ }^{13}$. Utilizing the Sutter Health EMR database in a cross section study for 2012 the analysis points to the importance of disaggregating chronic pain patients by pain type, identifying the number and types of pain conditions reported (with only $40 \%$ reporting one pain condition). At the same time account should be taken of the presence of comorbidities with only $28 \%$ of pain patients reporting no comorbidities while $21 \%$ report with 4 or more comorbidities the distribution of pain types.

Improving the quality of care can only occur if there is feedback to health care providers on the outcomes of treatment interventions within a clinically meaningful timeframe ${ }^{14}$. There is all too often a major disconnect between claims made for new products and devices to formulary committees and the ability of healthcare systems to validate such claims. Supporting feedback involves judging the credibility of claims made for pharmaceutical products and devices, evaluating these claims in treatment practice and providing the opportunity, as noted above, for claims replication across treating environments. This is achieved in the CPMR by requiring physician practices to detail reasons for therapy discontinuation, to include specific reasons for the discontinuation, by either the physician or the patient, of opioid therapy.

Outcomes claims should be judged in terms of clinical markers. The Food and Drug Administration (FDA) recognizes that in drug approvals evidence is required of a favorable benefit defined in terms of how a patient feels, functions and survives. It is important, therefore, as part of quality metrics to capture, via validated patient reported outcomes (PRO) instruments both response to therapy as well as measures that reflect symptoms and quality of life. This is addressed in the CPMR by asking patients, not only how their symptoms or comorbidities have improved (or at least been addressed) during the course of treatment but, utilizing the Patients' Global Impression of Change (PGIC) scale, how they would describe the change (if any) in activity limitations, symptoms, emotions and overall quality of life related to their painful condition since the beginning of their therapy. ${ }^{15}$ This question is asked at each follow-up visit to the practice. The seven responses allowed range from "No change (or condition got worse)", through "Somewhat better, but the change has not made any real difference" to "A great deal better, and a considerable improvement that has made all the difference". Care needs to be taken, however, in not focusing exclusively on the PGIC responses ${ }^{16}$.

The outcomes covered by the CPMR are also consistent with those proposed as core outcomes for chronic pain clinical trials by the Initiative on Methods, Measurement, and Pain Assessment in Clinical Trials (IMMPACT) ${ }^{17}$. The six core
IMMPACT domains are outcomes representing: (i) pain, (ii) physical functioning; (iii) emotional functioning; (iv) participant ratings of improvement and satisfaction with treatment; (v) symptoms and adverse events; and (vi) participant disposition (e.g., persistence/adherence). These domains are all reported in the CPMR.

\section{Chronic Pain Management Platform}

The CPMR incorporates 18 validated PRO instruments to capture response to therapy and comorbid behaviors. The CPMR captures from each patient:

- demographic and socio-economic characteristics

- overall pain experience

- prior chronic pain and the likelihood of continuing chronic pain

- pain experience and functional status for 13 designated pain locations

- medication utilization

- assessment of the likelihood of neuropathic pain

- indicators for possible fibromyalgia

- attitudes to pain medications

- opioid utilization

- risk for medication aberrant behavior

- self-reported aberrant medication behavior

- symptoms: fatigue, depression, anxiety, sleep

- constipation

- satisfaction with care

At the same time, the CPMR captures, in the provider version, the following as inputs from the practice:

- cause of pain (surgery, trauma)

- medication utilization

- ICD-10-CM diagnoses [CMS 1500]

- CPT (current procedures) [CMS 1500]

- adverse events [ICD-10-CM codes]

- specialty referrals

- urine test

- opioid use and consent

- medical marijuana use

- alternative therapy options

- reasons for therapy discontinuation

Combining these data elements, the CPMR creates a structured profile of therapy choice and therapy impact in chronic pain. This offers a unique insight into the clinical effectiveness of therapy in real-time with providers appraised at each visit whether patients are responding to therapy and whether the clinical response meets standards for a clinically meaningful improvement. At the same time, as detailed below, the CPMR allows the physician to focus on the choice of opioid therapy, the particular formulation selected and the response to therapy. Options for therapy switching are reported together with reasons for discontinuation. 


\section{CPMR Forms}

When a new patient joins a practice, the patient is typically asked to complete, either on-line through the practice CPMR portal or in paper form (downloaded from the CPMR), a series of forms. If paper forms are completed, these would be brought to the provider on first visit. If the CPMR is used, the selection of forms and the procedure for completion will be agreed with the practice. The CPMR includes, as a default, the following forms:

- Patient personal information (contact details, pharmacy, insurance, workers compensation, injury claims)

- Consent for treatment

- Privacy notice: Use and disclosure of medical information

- $\quad$ Practice financial policy (billing, insurance cover)

- Current prescription medications and over the counter products

- Authorization for use/disclosure of protected health information

As well, when required, the registry also includes two additional forms specific to chronic pain interventions. The first is a 'Patient Agreement: Pain Treatment with Opioid or Controlled Substance Medications' form that sets out the responsibilities of the patient and provider if opioids or other controlled substances are prescribed for long-term treatment of chronic pain. The second form is a 'Consent to Medical/Surgical Office Procedure' that may be part of the chronic pain treatment strategy. All forms require a patient signature. If a practice wishes to substitute or add its own forms this can be accommodated as part of a practice customization. Although the CPMR provides 'generic' forms, the practice may ask for its own forms to be substituted with additional forms if necessary.

\section{Patient CPMR Version}

Information requested from the patient and completed on line covers:

- Patient Initial Visit Report

- Patient Follow-up Visit Reports - follow-up reports for all subsequent practice visits (or requests for follow-up information in the absence of a practice visit)

- Patient Satisfaction Initial Visit Report (including pain management section)

- Patient Satisfaction Follow-up Visit Reports

On receipt of the initial visit report from the patient and the follow-up reports for all subsequent practice visits, a summary report is generated and transmitted directly to the pain practice so that it is available to the treating physician prior to the patient encounter. The follow-up visit summary reports link to the initial pain and functional status report to detail changes in pain and functional status since the first visit. This is supported by the patient's responses to the PGIC scale.

\section{Patient Initial Visit Report}

The Patient Initial Visit Report focuses on five areas. These are: (i) demographic and socio-economic characteristics; (ii) overall pain experience; (iii) pain and functional status by pain location; (iv) attitudes and risks in medication utilization; and ( $v$ ) symptoms and co-morbidities.

Evaluating outcomes of therapy, whether at the individual patient or the targeted patient group level, should take account of the impact of potential confounding factors. These factors may impact the ongoing experience of pain as well as the response of patients to therapy. In the CPMR the more prevalent comorbidities for non-cancer pain are captured in the validated instruments that capture the presence of fatigue, depression, anxiety, constipation and sleep. At the same time there are patient characteristics which should be considered. In particular: gender, race and ethnicity, employment status and education.

\section{Demographic and Socioeconomic Characteristics}

There is a substantive body of literature regarding sex difference in pain ${ }^{18}$. The evidence points to females reporting a higher prevalence and severity, together with increased pain sensitivity and risk for pain. At the same time, females differ in their response to pain treatment and in their perception of pain, although the evidence for the consistency of response to both pharmacological and non-pharmacological interventions is mixed. In the case of opioids, for example, the evidence suggests greater morphine analgesia for women and with greater analgesia than men for mixed action opioids. There is also evidence for gender biases in pain treatment.

Ethnicity is also a potential confounding factor in evaluating response to therapy. While there is no evidence that Hispanic and African-American status can impact response to therapy in pain management ethnicity may be a proxy for access to and compliance with care ${ }^{19} 20$.

Current employment status and the reasons for not working are also of interest in pain management. Of particular interest is the impact of pain on employment status and, if part of a pain management plan's objectives, the success of an intervention in returning a patient to work.

Educational attainment has long been recognized as a risk factor for chronic pain. Dionne et al, in a review of the relation between educational status and back pain published in 2001, concluded that the evidence suggests that rather than an association with onset, lower educational status is associated with longer duration and/or higher recurrence ${ }^{21}$. The likelihood of more disabling back pain is possibly explained by variations in behavioral and environmental risk factors, poor overall health, differences in occupational factors, differences in access and/or utilization and adaptation to stress. A Norwegian study, for example, found that each additional year of formal education was associated with a lower risk of a disability 
pension, while occupational class, working conditions and individual lifestyle reduced the effect of education ${ }^{22}$. Even so, there was still a 'strong and unexplained effect of education on back pain disability pensioning, which is not mediated by occupational class, working conditions or individual lifestyle'. These issues can be explored with data from the CPMR.

\section{Overall Pain Experience}

In large part because of the likelihood that a proportion of patients may report pain at more than one location together with the difficulty of establishing prior experience with pain levels and pain locations, the CPMR adopts a two stage approach in evaluating baseline pain experience at the initial visit.

In the first stage, patients are asked to detail their overall current pain experience together with their long-term experience of pain together and an assessment of their likelihood of their continuing to experience chronic pain. Current pain experience is defined in terms of (i) current pain on a standard visual analogue scale (VAS) where $0=$ no pain and 10 = pain as bad as it could be; (ii) worst pain (VAS scale) in past two weeks; and (iii) average pain (VAS scale) over the past two weeks. These scores are combined to give a 'pain intensity' score. Patients then report their prior experience of chronic pain and the impact of their pain experience in the past six months on their activity status utilizing the validated Chronic Grade Pain Scale (CGPS) instrument ${ }^{23}$. This yields two categories of pain and disability and two categories of disability and its limiting effects. Finally, in this section, the algorithm proposed by Von Korff and Dunn is applied, with supplementary questions, to yield a risk score for the likelihood of continuing chronic pain ${ }^{24}$.

\section{Pain and Functional Status by Pain Location}

In the second stage, the focus is on pain intensity and functional status by body location. The CPMR identifies 13 pain locations and uses, where feasible, validated instruments to assess baseline pain intensity and functional status, tracking this over the course of treatment. In some cases the instrument only identifies functional status and, where this occurs, supplementary questions to measure pain intensity are included. This format, as detailed below, is repeated for all subsequent visits (or reports) to create a tracking of patient response to therapy.

Table 1 details the 13 pain locations and the validated instrument that is proposed to assess functional status and, unless supplemented by a pain intensity score, the pain level reported. Scores are presented in the patient summary and sent to the provider both for the aggregate score as well as scores categorized to grade levels and subscales. Patients indicate which pain location (or locations) is (are) appropriate and a drop down screen is presented for the instrument completion together with supplemental pain intensity questions if required. As patients are asked to respond to their nominated pain locations at each subsequent visit, the instruments and supplemental pain questions create a realtime profile of response to therapy for both pain intensity and functionality.

The CPMR also addresses the question of the likelihood of the patient experiencing neuropathic pain. Although a clinical diagnosis for neuropathic pain is the 'gold standard' a number of instruments have been proposed as symptom-based screening questionnaires for neuropathic pain. These instruments include the Self-completed Douleur Neuropathique 4 (S-DN4) ${ }^{25}$, the ID-Pain ${ }^{26}$, the pain detect questionnaire (PDQ) 27 and the Self-completed Leeds Assessment of Neuropathic Symptoms and Signs (S-LANSS) ${ }^{28}$. For the purpose of the CPMR, the instrument selected was the ID-Pain with 6 sensory description items. The ID-Pain is short, it does not involve any patient self-examination and psychometrically it performs as well as the S-DN4 and PDG, and better than the S-LANSS ${ }^{29}$. Similarly, in the case of possible fibromyalgia, the patient is asked to report on the pain experience across multiple locations. Again this is only indicative and is not a substitute for a clinical assessment.

It is worth emphasizing again that the client has the option of customizing their version of the CPMR to include alternative pain and functional status instruments. The Oswestry scale could, for example, be substituted for the Roland Morris Disability instrument scale with alternative instruments for head pain and lower extremity pain. If substitutions are required then it is the responsibility of the client to obtain the necessary permissions and pay any fees if not in the public domain.

In the case of scores for hip pain, leg pain and foot or heel pain the assessment is in terms of (i) response to an item list (13) to give assessment of status by item but no overall score and (ii) pain intensity at present time, worst in previous two weeks and average over past two weeks. Again, there is the option for a client to choose a specific measures of pain and functional status for hip, leg or foot/heel pain (e.g., Oxford Hip Score ${ }^{30}$ ) 
Table 1

Chronic Pain Management Platform: Pain Locations and Validated Instruments for Assessing Pain and Functional Status

\begin{tabular}{|c|c|c|}
\hline Pain Location & Instrument & Score \\
\hline Head Pain & $\begin{array}{l}\text { Migraine Disability Assessment Score } \\
\text { (MIDAS) }{ }^{31}\end{array}$ & $\begin{array}{l}\text { Grade 1: Little or no disability (score } 0-5 \text { ) } \\
\text { Grade 2. Mild disability (score 6-10) } \\
\text { Grade 3: Moderate disability (score } 11-20 \text { ) } \\
\text { Grade 4: Severe disability (score } 21+\text { ) }\end{array}$ \\
\hline Orofacial Pain & $\begin{array}{l}\text { Manchester Orofacial Pain and Disability } \\
\text { Scale }{ }^{32}\end{array}$ & $\begin{array}{l}\text { Physical Disability (score 0-14; } 0=\text { least disability) } \\
\text { Psychosocial Disability (score 0-38; } 0=\text { least } \\
\text { disability) } \\
\text { Aggregate (score } 0-52 \text { ) }\end{array}$ \\
\hline Neck or Cervical Pain & $\begin{array}{l}\text { Copenhagen Neck Functional Disability } \\
\text { Scale }{ }^{33}\end{array}$ & $\begin{array}{l}\text { Aggregate score }(0-30 ; 0=\text { no real complaints to } \\
30=\text { real disability })\end{array}$ \\
\hline Shoulder Pain & $\begin{array}{l}\text { Shoulder Pain and Disability Index } \\
\text { (SPADI) }\end{array}$ & $\begin{array}{l}\text { Aggregate score, total pain score and total } \\
\text { disability score each as a percentage. Minimum } \\
\text { detectable change } 13 \text { points ( } 90 \% \text { confidence) }\end{array}$ \\
\hline Wrist or Hand Pain & $\begin{array}{l}\text { Patient- Rated Wrist Hand Evaluation } \\
\text { (PRWHE) }\end{array}$ & $\begin{array}{l}\text { Pain score }(0=50) \text {, Function score }(0-50) \text { and total } \\
\text { score }(0=\text { best score; } 100=\text { worst score })\end{array}$ \\
\hline Mid-back or Thoracic Pain & Roland Morris Disability Scale ${ }^{3738}$ & Score $0-24$ (score $\geq$ poor outcome) \\
\hline Lower-back or Lumbar Pain & Roland Morris Disability Scale & Score $0-24 ;$ (score $\geq$ poor outcome) \\
\hline Hip Pain & Items only & No validated aggregate score or subscales \\
\hline $\begin{array}{l}\text { Lower Abdominal Pain } \\
\text { (Males) }\end{array}$ & $\begin{array}{l}\text { NIH-CPSI Abdominal Pain } \\
\text { Questionnaire }\end{array}$ & $\begin{array}{l}\text { Pain: score } 0-21(0=\text { least pain }) \\
\text { Urinary Symptoms: score } 0-10(0=\text { no problem }) \\
\text { Quality of Life: score } 0-12(0=\text { no impact })\end{array}$ \\
\hline $\begin{array}{l}\text { Lower Abdominal Pain } \\
\text { (Females) }\end{array}$ & NIH-CPSI Abdominal Pain Questionnaire & $\begin{array}{l}\text { Pain: score } 0-21(0=\text { least pain }) \\
\text { Urinary Symptoms: score } 0-10(0=\text { no problem }) \\
\text { Quality of Life: score } 0-12(0=\text { no impact })\end{array}$ \\
\hline
\end{tabular}

\section{Attitudes and Risks in Medication Utilization}

A particular focus of the CPMR is not only on the outcomes experienced by patients treated with opioid medications, but on patient experience with medications, their attitudes to pain medications, the likelihood of medication abuse and a selfreport of actual 'abuse' reported by patients. The CPMR captures, in the initial patient report, medication utilization, patient experience with opioids (to include prior opioid prescriptions and opioid use within the past 30 days), patient attitudes to pain medications (see below) and an assessment of likelihood of aberrant medication behavior. In the follow-up reports by patients (see below) a series of questions on aberrant medication use behavior is presented to capture the extent of such behaviors, The categorization of opioids for self-reporting is presented in Table 2. More precise details on medication utilization can be generated in the provider/patient registry version from the physician office report to include brand name (NDC code), dosage, number of tablets and days supplied (see below). This provides a more accurate picture of medication use as opposed to asking patients to self-report medication use. 
Table 2

Chronic Pain Management Platform: Opioid Categorization

\begin{tabular}{|l|}
\hline Opioid Category \\
\hline Oxycodone (e.g., Oxycontin, Roxicodone, Oxecta, Percocet, Percodan, Xtampza, Targiniq, Troxyca, Roxybond) \\
\hline Hydrocodone (e.g., Anexia, Hycet, Vicodin, Lorcet, Lortab, Vicodin, Zydone, Hyslinga, Vantrela) \\
\hline Morphine (e.g., MS Contin, Oramorph SR, Avinza, Arymo, Morphabond, Embeda) \\
\hline Hydromorphone (e.g., Dilaudid, Exalgo, Palladone) \\
\hline Fentanyl (e.g. Actiq, Fentora, Duragesic, Subsys, Sublimaze, Abstral, Onsolis, Lazanda) \\
\hline Codeine (e.g., Tylenol with codeine) \\
\hline Methadone (e.g., Dolophine, Methadose) \\
\hline Meperidene (e.g., Demerol) \\
\hline Oxymorphone (e.g., Opana) \\
\hline Tramadol (e.g., ConZip, Ultram, Ryzolt) \\
\hline Tramadol (e.g., ConZip, Ultram, Ryzolt) \\
\hline $\begin{array}{l}\text { Buprenorphine (e.g., Subutex, Buprenex, Butrans, and Probuphine and Buprenorphine/Naloxone combinations } \\
\text { Suboxone, Zubsolv, Bunavail) }\end{array}$ \\
\hline
\end{tabular}

Note: Self-reported for (i) any previous prescription (no time limit) and (ii) use in last 30 days.

A number of validated instruments have been developed to assess patients' attitude to medications, to include their beliefs and concerns, as these have the potential to impact medication choice, utilization, adherence and persistence. In 2006 McCracken el al reported on the development of a 78-item Pain Medication Attitude Questionnaire (PMAQ) that addressed seven areas of patient concern: addiction, perceived need, unfavorable scrutiny by others, adverse side effects, tolerance, mistrust in the prescribing doctor and withdrawal ${ }^{41}$. More recently Elander et al have proposed a short form 14-item version (PMAQ-14) of the original questionnaire, capturing from the original 2 items from each of the seven PMAQ scales those that retained the content of the original scales ${ }^{42}$. Confirmatory factor analysis demonstrated that the PMAQ-14 retained the original 7-factor structure with correlation analysis demonstrating that it retained the validity of the original constructs. The PMAQ-14 was chosen for the CPMR.

As well as capturing attitudes to medications, the CPMR also addresses the question of the risk for prospective medication aberrant behavior. Once again, a number of risk assessment instruments have been proposed. These include the Pain Medication Questionnaire (PMQ), the Screener and Opioid Assessment for Patients with Pain - Revised (SOAPP-R; 5, 14 and 24 item versions), the Opioid Risk Tool (ORT) and, most recently, the Brief Risk Questionnaire (BRQ) ${ }^{4344466}$.

The BRQ was chosen in preference to other risk assessment instruments, specifically the Opioid Risk Tool (ORT) and the Screener and Opioid Assessment for Patients with Pain - Revised (SOAPP-R), because, on evidence available it is overall the most accurate with a sensitivity of $80 \%$ and a specificity of $41 \%$. Sensitivity in this context is the ability to identify those patients who later were observed to engage in opioid or medication misuse. The sensitivity of the $B R Q$, which is best seen not as a 12-item overall assessment of risk but as an evaluation of 12 areas of risk, also compared favorably in a head-to-head comparison with the structured clinical Brief Risk Interview (BRI) ${ }^{47}$.

Although outside the scope of the registry at this time, as it involves both patient reporting and clinical assessment, the BRI is an accepted 'gold standard' for the various PRO instruments with, in this comparison, a sensitivity of $79 \%$ and specificity of $51 \%$. The sensitivity of the ORT instrument was found to be $32 \%$ with a corresponding specificity of $82 \%$; the corresponding figures for the SOAPP-R instrument were $33 \%$ and $77 \%$. While the specificity of the ORT and SOAPP-R instruments was higher, it is worth noting that the ability to detect patients unlikely to engage in medication misuse should be set against the higher costs to the patient and society of misuse so that, on balance, a false positive understatement of the likelihood of appropriate behavior is acceptable.

\section{Symptoms and Co-morbidities}

The final section of the initial patient questionnaire provides a baseline status for five comorbidities or symptoms associated with chronic pain: fatigue, depression, anxiety, constipation and sleep. All the instruments used have been validated. The details are given in Table 3 . These are not the only comorbidities and adverse events (side effects) that are captured by the CPMR. The physician practice (see below) is asked to report on other diagnosed comorbidities (ICD-10-CM codes) and adverse events (ICD-10-CM codes).

One reason for selecting these comorbidities and symptoms (e.g., presence of constipation with opioid use and its treatment) is that it is important, not only to attempt to assess the impact 
of treatment interventions on pain and functional status but the association with the alleviation of fatigue, depression anxiety and sleep. Constipation is, of course, a well- documented side effect of opioid medications and one that while it can be treated may impact the willingness to continue therapy

\section{Patient Follow-Up Visit Reports}

For all subsequent visits to the physician practice over the course of treatment, patients are requested on-line to complete a Patient Follow-up Visit Report prior to their practice visit. This can be programmed to ask patients to complete follow-up reports even if they do not attend the practice; the schedule to be determined by the treating physician. The follow-up report captures all information covered in the initial visit report with the exception of: (i) socio-demographic characteristics; (ii) prior chronic pain experience and the likelihood of continuing chronic pain; and (iii) attitude to medications and the risk of aberrant medication behavior. Additional information requested in the self-reports are: (i) pain medication ADRBs (aberrant drugrelated behaviors) in the past 30 days or since last practice visit; and (ii) patient perception of change applying the PGIC instrument.

Table 3

Chronic Pain Management Platform: Symptom and Co-Morbidity Instruments

\begin{tabular}{|c|c|c|}
\hline Symptom or Comorbidity & Comorbidities and Symptoms & Score \\
\hline Fatigue & Fatigue Severity Scale 4849 & $\begin{array}{l}\text { Single score as average of } 9 \text { items; presence } \\
\text { of fatigue } \geq 4 \text {. Note: mean } 2.3 \text { (SD +/- } 0.7 \text { in } \\
\text { normal healthy adults) }\end{array}$ \\
\hline Depression & PHQ-9 Depression Scale ${ }^{50}$ & $\begin{array}{l}\text { Score range } 0-27 \\
\text { Minimal Depression (score } 0-4 \text { ) } \\
\text { Mild Depression (score } 5-9 \text { ) } \\
\text { Moderate Depression (score } 10-14 \text { ) } \\
\text { Moderately severe depression (score } 15-19 \text { ) } \\
\text { Severe Depression (score } 20+\text { ) }\end{array}$ \\
\hline Anxiety & GAD-7 Anxiety Scale ${ }^{51}$ & $\begin{array}{l}\text { Score range } 0-28 \\
\text { None or very mild anxiety (score } 0-4) \\
\text { Mild anxiety (score } 5-9) \\
\text { Moderate anxiety }(10-15) \\
\text { Severe anxiety }(16+)\end{array}$ \\
\hline Constipation & PAC-SYM Questionnaire ${ }^{52}$ & $\begin{array}{l}\text { Score averaged over } 11 \text { items (range } 0-4 \text { ) } \\
\text { Average or mild constipation (score } 0-0.9 \text { ) } \\
\text { Moderate constipation (score } 1.0-2.9 \\
\text { Severe or very severe constipation (Score } \\
3.0+\text { ) }\end{array}$ \\
\hline Sleep & $\begin{array}{l}\text { RAND Sleep Report and } \\
\text { Questionnaire }{ }^{53}\end{array}$ & $\begin{array}{l}\text { Sleep Experience (last } 2 \text { weeks): Time taken } \\
\text { to fall asleep, hours of sleep. RAND Sleep } \\
\text { (score } 0-50 ; 0=\text { no problems to } 50 \text { extreme } \\
\text { problems) }\end{array}$ \\
\hline
\end{tabular}

There are a number of instruments to choose from when it comes to identifying those patients with chronic pain taking opioids (or other medications) who have indications of current opioid risk and aberrant drug-related behaviors. As the CPMR is designed, not for one-off measures of ADRBs, but for regular patient assessments, the instrument should ideally be one that interrogates patients for behavior since their last visit or within a relatively short time frame. Given this constraint, the Prescription Drug Use Questionnaire - patient version (PDUQp) is clearly unsuitable as the question time frames are either open ended or refer to the previous 6 months ${ }^{54}$. The PDUQp is also unacceptable given the possibility of respondent fatigue given the 31 items that comprise the questionnaire.

Another possible candidate is a modified shorter version of the PMQ comprising 10 items from the PMQ and a further 5 items covering selling, forging prescriptions, internet sources and using medications involving crushing and snorting 55 . Consideration was also given to utilizing the Current Opioid Misuse Measure (COMM) ${ }^{56}$. This is a 17 item validated measure 
to identify patients with chronic pain who have indicators of current ADRBs. The COMM utilizes Likert scales $(0-4)$ with a cut-off score of 9 points. However, to avoid possible conflicts as the COMM is already used with the SOAPP-R as an on-line commercial product tool for assessing opioid behavior, it was decided to generate a shorter item set (to minimize respondent fatigue) which covered the current aberrant behaviors commonly identified in these various instruments with a yes/no response. No attempt was made to provide an item response score to try to evaluate the 'severity' of the aberrant behavior. That is left to the treating physician.

The items are:

- In the past 30 days or since your last visit to this practice have you lost or misplaced your pain medication and had to ask for a replacement?

- In the past 30 days or since your last visit to this practice have you sold any of your pain medication?

- In the past 30 days or since your last visit to this practice are there days when you have taken more of your pain medication than prescribed?

- In the past 30 days or since your last visit to this practice have family members or friends commented on how you were dealing with you pain medication?

- In the past 30 days or since your last visit to this practice have you forged a prescription in order to obtain pain medication?

- In the past 30 days or since your last visit to this practice have family members or friends obtained pain medication for you (e.g., from their physicians)?

- In the past 30 days or since your last visit to this practice have you tried to supplement your pain medication(s) with other drugs to help your pain medication work better?

- In the past 30 days or since your last visit to this practice have you obtained pain medications from the internet or other non-medical source?

- In the past 30 days or since your last visit to this practice have you used your pain medication in a form other than how it was prescribed (e.g., crushed, snorted, injected)?

- In the past 30 days or since your last visit to this practice have you visited another physician, including urgent care, to obtain pain medication?

- In the past 30 days or since your last visit to this practice have you visited the emergency room to obtain pain medication?

- In the past 30 days or since your last visit to this practice have you taken pain medications belonging to someone else?

- In the past 30 days or since your last visit to this practice have you used your pain medications for conditions other than your pain (e.g., to help you sleep, to relieve stress)?

\section{Patient Satisfaction Initial Visit Report}

Following the first practice visit, the patient is asked to complete on-line an Initial Patient Satisfaction and Pain Management Assessment Report. For all subsequent visits the patient only completes the patient satisfaction with care component. This covers:

- Provider: satisfaction with care provided (thoroughness, time spent, overall quality of care)

- Other Staff: satisfaction with care provided (reception staff, courtesy)

- $\quad$ Practice access: satisfaction with access provided ( contacting office, waiting in office, parking)

Where, in each case, responses are on a five point scale: excellent, good, just satisfactory, fair or poor.

In addition, the patient completes after the initial visit as part of the satisfaction report a series of questions on the extent to which a pain management plan has been provided and whether specific issues (e.g., fatigue, anxiety, depression, sleep) have been addressed. The patient is told that: A pain management plan is a proposal for how your pain problem is to be addressed; it might involve a proposal for a management team involving other specialist providers and nursing support, treatment options and possible use of pharmaceuticals, a timeline for treatment and the outcomes sought. You might also be advised on the potential for over dosing and abuse of prescription pain medications.

The specific questions are:

- $\quad$ Did your provider discuss and agree a pain management plan with you?

- Did you provider review your past medication use in the treatment of your pain condition?

- Did your provider discuss the benefits and risks of the use of opioids in the treatment of your pain?

- Did your provider discuss the benefits and risks of prescribing 'risk abuse' opioid formulations?

- Did your provider discuss the possibility of non-opioid drug formulations in the treatment of your pain?

- Did your provider discuss how long you might be on pain medication?

- Did your provider discuss what non-pharmacological options there might be for your pain condition (e.g., surgery, acupuncture)?

- Did your provider discuss how any comorbid disease states that you have (e.g., cardiovascular disease, diabetes) might impact your pain treatment?

- Did your provider discuss the possible involvement of other health professionals in your treatment?

- Did your provider assess and recommend treatment for fatigue?

- Did your provider assess and recommend treatment for anxiety? 
- Did your provider assess and recommend treatment for depression?

- Did your provider assess and recommend treatment for constipation?

- Did your provider discuss what the side effects of your treatment might be?

- If you are not at work, did you discuss with your provider the possibility of returning to work?

\section{Patient Summary Reports to the Physician Practice}

There are two patient response summary reports prepared automatically by the CPMR and transmitted to the physician practice for each patient prior to the patient's encounter with the provider. These are

- Patient Initial Visit Summary Report

- Patient Follow-up Visit Summary Report

All summary reports provide individual item scores as well as summary scores for validated instruments. The summary report to the physician or practice includes:

- Demographic and sociodemographic responses

- Present and previous health status

- Present pain experience (pain intensity)

- Pain experience and impact in last 6 months

- Chronic Graded Pain Scale

- Likelihood scores for continuing chronic pain

- Pain location(s)

- Pain status and functionality scores by pain location

- Possibility of neuropathic pain/fibromyalgia

- Prescription opioid history

- Opioid use in past 30 days

- Views on pain medications (scores for addiction, need, scrutiny, side effects, tolerance, mistrust of doctors and withdrawal concerns)

- Aberrant opioid risk assessment

- Fatigue score

- Depression score

- Anxiety score

- Constipation score

- $\quad$ Sleep experience (last two weeks) and sleep problems score

If the provider is linked to a medical marijuana dispensary for the patient, then following each dispensary visit the provider will be sent:

- Cannabis administration form and dosing

- Titration (if necessary)

- Pain intensity and pain functional status by location change over baseline

- Comorbid status and change over baseline

- PGIC
- Change in other conditions if reported (severe nausea, persistent muscle spasms, seizures)

The Patient Follow-up Visit Summary Reports provide a summary of current status versus initial or baseline status over the course of treatment for:

- Employment status

- Overall health

- Pain experience

- Pain status and functionality score by pain location

- Item checklist for aberrant medication behaviors

- Fatigue

- Depression

- Anxiety

- Constipation

- Sleep experience

- Patients' Global Impression of Change (PGIC)

Each Patient Follow-up Visit Summary report also includes an item checklist for aberrant medication behaviors (see above).

To assist the physician assessing the statistical and clinical significance of changes in the various scores, notes are included in the summary report to the physician with estimates of (i) minimum detectable change (MDC) and (ii) minimum clinically important change (MCID). The MDC is a statistical estimate of the smallest amount of change that can be detected by a measure that corresponds to a noticeable change that is not attributable to measurement error (estimated at $90 \%$ or $95 \%$ confidence intervals). The MDIC takes the physicians' perspective in judging whether a change is clinically significant or, put differently, the minimum amount of change that a physician judges is required for a patient to feel a difference. The patient may not, of course, concur in this assessment of change. For this reason the PGIC scale is also reported.

\section{Patient and Provider CPMR Version}

The provider version of the CPMR requires the practice to report on the individual patient. Subsequent to or during each practice encounter with the patient, the practice is asked to submit online a Physician Office Visit Report. There is a separate report for the initial patient visit, the Physician Office Initial Visit Report and a standard report for all follow-up visits, the Physician Office Follow-up Visit Report.

\section{Physician Office Visit Reports}

The Physician Office Initial Visit Report and the Physician Office Follow-up Visit Report are designed to capture a range of information that has been collected by the practice as part of the patient encounter. Specific data elements are captured from those reported on the CMS 1500 form or its EMR equivalent (paper or electronic) in respect of the ICD-10-CM diagnosis codes and CPT codes. These are supported by questions on: (i) pain evaluation; (ii) medication utilization; (iii) adverse events; 
(iv) opioid experience and options; and (v) discontinuation of therapy.

The pain evaluation questions ask, first, whether the pain is the result of trauma or injury (e.g., personal injury at home, motor vehicle accident, military service). If this is not the case, the practice is then asked to detail whether it was caused by surgery (which may be related to trauma or injury) with the type of surgery to be detailed by date of surgery, CPT procedure code and a brief description. If the pain is not related to trauma, injury or surgery, the practice is then asked to report on whether it is related to a comorbid condition (e.g., diabetic neuropathy) defined by the appropriate ICD-10-CM code.

The practice is asked to detail all prescription medications currently reported. Comparing these over time within the registry framework will give a profile of use, switching, dosage change and discontinuation. . For each medication the practice is asked to list: generic and brand name, dosage, number of tablets/pills per day, days supplied, whether the medication is being titrated or is a rescue medication, together with the NDC code.

Apart from prescription medications, the physician's office is also asked to report if the patient has been recommended for medical marijuana. If the patient is reported as having met certification requirements, then the office is asked to report the $\mathrm{THC} / \mathrm{CBD}$ (or CBD) formulation and mode of administration.

It is important that in reporting ICD-10-CM diagnoses that the entries capture both the primary diagnosis as well as any supplementaryor comorbid diagnoses (e.g., diabetes, cardiovascular disease, depression, anxiety) using billable codes. Practices are asked to be as precise as possible in indicating the type of pain and to avoid the use of non-specific or nor billable pain codes. Importantly, the ICD-10-CM codes reported should match the pain locations identified by the patient.

As an example, as functional status often refers to a joint and the presence of joint pain, the use of only billable codes would exclude the use of M25.5 Pain Joint (non-billable) or at a lower level M25.51 Pain in Shoulder (non-billable) but report more specifically the billable code M25.511 Pain in Right Shoulder. Although a billable code, the code M25.50 Pain in Unspecified Joint should not be used. This level of detail does not apply, for example to M54.6 Pain in Thoracic Spine which is a billable.

The practice is then asked to detail whether or not an office procedure was performed. A list of interventional procedures is given with the practice asked to check off the particular intervention, with a write in response for a non-Olisted intervention. The practice is not asked to report CPT/HCPS codes. Future versions of the CPMR might consider these to give more granularity but at a possible added burden on the practice.
The practice is asked also to report adverse events. It is up to the practice to determine the severity and appropriateness of reporting. When these events are reported the appropriate ICD$10-\mathrm{CM}$ code should be used. It should be noted that these may duplicate billable ICD-10-CM codes that may already have been entered (e.g., K59.00 Constipation, unspecified, K59.04 Chronic idiopathic constipation, K50.09 other constipation, R11.2 Nausea with vomiting, unspecified).

Given the likelihood of significant comorbidities accompanying a diagnosis of chronic pain, the practice is also asked whether or not referrals have been made for assessment/treatment by a specialist. The items of interest are (i) specialty to which the patient has been referred and (ii) the reason for referral. Importantly, the provider is also asked to report whether the patient has been recommended or certified for medical marijuana. If certification has occurred, the provider is asked to detail the dispensary providing the access to botanical cannabis. This provides the link for the dispensary to submit visit reports on the patient's status in respect of chronic pain and any associated conditions (e.g., severe nausea). If the dispensary has adopted the associated registry platform, the outcomes reported will be consistent with those tracked by the provider practice both prior to the recommendation for medical marijuana and any ongoing interaction with the provider if the cannabis dispensed, for example, is part of a chronic pain polytherapy regimen.

The physician's office is asked to report in detail on the patient's experience with opioids and efforts by the practice to reduce exposure to opioids. The key data elements requested are (i) urine testing; (ii) presence of an opioid agreement; (iii) referral to a methadone/buprenorphine treatment program; (iv) tapering/discontinuation of opioid therapy; and ( $v$ ) offers of alternative pain management interventions.

Under (iv) the practice is asked to report the reason(s) for tapering/discontinuation of opioid therapy. The options are:

- Failure to show sufficient analgesia

- Failure to show functional improvement

- Excessive dosing to achieve pain management targets

- $\quad$ Presence of significant clinical risk factors (e.g., sleep apnea)

- Impact of medication side/adverse effects

- Patient request

- Patient's family request

- Opioid aberrant behavior

- Non-opioid substance use aberrant behavior

- Failure to agree to adopting a non-opioid pain management plan

As the CPMR also tracks botanical cannabis use (including, it should be noted, TCH/CBD combinations and non-hemp derived $\mathrm{CBD}$ products) the questions to the opioid 
tapering/discontinuation can be applied to both hemp-derived and non-hemp marijuana products.

Finally the practice is asked to report on the scheduling of the next appointment and, where a future appointment has not been scheduled the reason why it has not been scheduled. The practice is asked, from a check list, to give the reason(s) for nonscheduling. The options include satisfactory resolution of pain and functional symptom, patient decision to cease treatment even if symptoms not resolved, unacceptable side effects and moving to another practice.

\section{Practice Standardized Patient Reports}

The CPMR, in the patient/provider version, generates at the end of each month seven practice summary reports. Modified versions are available for the patient only CPMR version. These reports are:

(i) Monthly Practice Profile Initial Encounter Report

(ii) Monthly Practice Profile Initial Pain Intensity and Functional Status Report

(iii) Monthly Initial Encounter Satisfaction Profile

(iv) Monthly Pain Location Continuing Patients Outcomes Report

(v) Monthly Procedures Continuing Patients Outcomes Report

(vi) Monthly Continuing Patients Pain Location and Opioid Outcomes Report

(vii) Monthly Continuing Patients Opioid Outcomes Report

\section{Monthly Practice Profile Initial Encounter Report}

This report details for all patients who were initially enrolled in the CPMR in that month the key data elements reported in the Patient Initial Visit Report and the Patient Initial Visit Summary Report. It excludes functional status by pain location which is detailed in the second monthly practice profile report (below).

\section{Monthly Practice Profile Initial Pain Intensity and Functional} Status Report

The report focuses on the pain locations reported by patients at their initial visit and gives a summary across all patients the average pain intensity and functional status reported. This is the baseline for subsequent assessments of the impact of therapy interventions by pain location.

\section{Monthly Pain Location Outcomes Report}

The monthly pain location outcomes report is for continuing patients and can be modified for time elapsed since initial therapy intervention. Standard timeframes are 90, 180 and 270 days.
Monthly Initial Encounter Satisfaction Profile

For each satisfaction with care item the number of patients and the average score (range $0-4$ ). For initial patients there is a further list of items that a provider might have considered as an element of a pain management plan. The numbers of responses to each question are given with percentage of total responses.

Monthly Pain Location Continuing Patients Outcomes Report This report captures at the end of each month the outcomes reported by patients who have maintained adherence to their initial pharmacotherapy, including dosage adjustments and supplemental pharmacotherapy. Initial number of patients are recorded, the number who have discontinued therapy, the number who have received modified pharmacotherapy and their responses to the PGIC scale, This gives a distribution of responses from 'no change' to 'a great deal better'. The time frame for the response is adjusted to 90, 180 and 270 days, Responses are given for each pain location treated.

\section{Monthly Procedures Continuing Patients Outcomes Report}

This report is identical to the previous report in the distribution of PGIC responses by the type of interventional procedure. Patients are identified if they have received supplemental pharmacotherapy with a similar timeframe options for reporting.

\section{Monthly Continuing Patients Pain Location and Opioid Report} This report is identical to the first pharmacotherapy report while restricted to patients who have taken opioids.

\section{Monthly Continuing Patients Opioid Outcomes Report}

The focus of this report is on the medium to long-term response to opioids in chronic pain. The table details for each of the opioid classes the number of patients initiated to that class of opioids, the number who have dropped out from therapy and the number receiving modified or supplementary pharmacotherapy. For continuing patients at 90, 180 and 270 days the response to therapy is captured by the PGIC scale.

The point to note is that these standard monthly reports provide a framework for a possible more in-depth assessment of the response to therapy by target patient groups. In the case of those initiated to an opioid therapy (e.g., a recently introduced abuse deterrent formulation) the practice might be interested in the response to individual products within an opioid class and those patients who, at 90 days, report no change in their response to the intervention. This could be supplanted by reasons for opioid therapy discontinuation and the possible impact of medication abuse behaviors.

Once a pain location is selected, the CPMR can specify the pharmacology (e.g., Oxycodone or Xtampza ER as a specific product) or intervention used (e.g., Genicular Nerve Ablation) with tables created to compare various interventions (e.g., 
comparing abuse deterrent opioid formulations). Alternatively, the CPMR can generate tables for all patients initially treated for lower back pain without further detail for individual interventions. Finally, the CPMR can specify the time since initial therapy (initial visit date). The report will capture all patients in the CPMR that meet these criteria and seek their input to table creation.

\section{Meeting CPMR Reporting Requirements}

A perennial concern in treatment practice is the question of the resources available to streamline workflow as well as endeavoring to meet demands placed on the practice for meeting quality metrics and, in the case of the CPMR, meeting the reporting standards. In the last case the resource demands are (i) allocating time to evaluating and reporting on the patient in completing the post-visit reports and (ii) assigning responsibilities for evaluating and reporting on the standard monthly reports from the CPMR as well as generating customized reports for the practice.

Understandably, physician practices are reluctant to introduce reporting standards where a practice is already overworked in terms of the time allocated to patient assessment and the additional resources that may be required to evaluate practice performance in the treatment of chronic pain. In short, where practice resources are scarce and overstretched, there has to be an incentive for practices to adopt quality metrics and engage in a more comprehensive assessment of practice performance. This is of particular importance in the management of chronic pain where the societal costs of opioid misuse and abuse are substantial with physician practices, in particular specialized pain practices, being asked to shoulder greater responsibilities.

It is not the intention to offer advice on the possibility and practicability of attracting additional financial resources to the practice through the various elements (e.g., pain and functional status assessments, opioid risk and abuse evaluations) captured on an ongoing basis by the CPMR. Even so, it is worth noting potential opportunities to augment practice resources, including covering the operational costs of the CPMR and generating additional revenue to support ongoing pain management.

One example is the potential for supplementing practice revenue through meeting the various requirements of the Merit Based Incentive Payment System (MIPS) score: quality measures (2018: 50\%), advancing care information (2018: 25\%), improvement activities (2018: 15\%) and resource use (2018: $10 \%$, calculated by CMS). In the case of quality measures, the CPMR captures a substantial number of the proposed metrics. These include metrics for functional status change (e.g., ID 182, ID 217, ID 218, ID 219, ID 220, ID 221, ID 222, ID223) as well as more general metrics such as ID 131 Pain Assessment and Follow-up, metrics relating to opioid assessment and use (ID
412, ID 414, ID 408) and those for depression screening and follow up (ID370, ID 371, ID 411) ${ }^{57}$.

In the case of advancing care information the CPMR supports reporting on the baseline performance measures (e.g., health information exchange) with additional points possible for reporting data to a platform, advance care planning and promoting the use of patient-reported outcomes tools ${ }^{58}$.

Finally, under improvement activities the CPMR captures a number that may qualify. These include Activity 1A_EPA_2 Collection and use of patient experience and satisfaction data on access, Activity 1A_PM_14 Implementation of methodologies for improvements in longitudinal care management in high risk patients and activity $1 \mathrm{~A} \_B E_{-} 4$ Engagement of patients through implementation of improvements in patient portal ${ }^{59}$.

Apart from MIPS, there is the possibility of utilizing CPT billable codes to capture the data and information elements covered by the CPMR. Before considering this opportunity advice should be sought from coding and billing experts. It is not the role of this description of the CPMR to offer advice or to encourage this option. Exercising this option is at the discretion of the physician practice. Reimbursement rates may vary depending on practice location and insurance cover, with commercial insurers reimbursing at different rates from Medicare and Medicaid, including the number of visits made to the practice with codes applicable and potentially reimbursed at each visit. These billable codes potentially include, to give a few examples, CPT 96127 for scoring and documenting brief emotional and behavioral assessments/screening codes (four per patient visit); CPT 96103 computer administered psychological testing: CPT 99490 chronic care management, together with the more time consuming CPT 99487 and CPT 99489 codes; CРT96160 for health risk assessment; and G-codes for alcohol and/or substance abuse screening and intervention and associated $G$ codes for functioning and physical therapy. Although not pain management specific, there are a range of evaluation and management CPT codes that should be considered with the information generated by the CPMR. These cover: (i) new patient visit (CPT 99201 to 99205) and (ii) established patient visit CPT 99211 to 99215). For a more comprehensive approach to coding in pain management there are the web site and code books of the American Academy of Pain Management ${ }^{60}$.

\section{Conclusions}

Practice based registries with inputs from both patients and providers are not new. What sets the CPMR apart is the focus on the management of chronic pain with real-time feedback to both patients and providers. The treatment of chronic pain in the US is a particular concern: opioids are abused and physicians have little guidance on the benefits and risks in the medium and long term. While they may be reluctant to prescribe opioids, they are also faced with a dearth of alternatives. Botanical cannabis may 
be one solution, but as in the case of opioids, evidence is required to support decision making.

Where resources are scarce, the application of validated PROs and other measures of response to therapy can potentially support provider claims for additional resources. These may be through meeting quality metric standards as well as from billable codes for reimbursement. Whether these possibilities are acted on is a decision for the practice. The point is, however, that they provide a basis for additional practice resources to enhance the process and duty of care in chronic pain.

A final point to note is the proposed platform can be implemented in stages. An initial installation could be in terms of just patient reporting with reports sent to the practice at scheduled visits. This version would still support billable codes as these are based on the patient reported section of the complete platform. A practice may then decide to implement provider reporting as well as patient reporting. This would include patterns of adherence, reasons given by the provider for therapy switching and therapy termination.

Conflicts of Interest: None declared 


\section{References}

${ }^{1}$ Schommer JC, Isetts BJ. Greater expectations for pharmaceutical value: Better care, healthier people, smarter spending. Inov Pharm. 2016, 7(2): Article 8

2 Dowell D, Haegerich TM, Chou R. CDC Guideline for Prescribing Opioids for Chronic Pain - United States, 2016. MMWR Recomm Rep. 2016; ePub: March 2016: DOI: http://dx.doi.org/10.15585/mmwr.rr6501e1er

${ }^{3}$ Simopoulos T, Manchikanti L, Gupta S et al. Systematic Review of the diagnostic accuracy and therapeutic effectiveness of sacroiliac joint interventions. Pain Physician. 2015;18(5):E13-56

${ }^{4}$ Davis T, Loudermilk E, DePalma M et al. Prospective, multicenter, randomized, crossover clinical trial comparing the safety and effectiveness of cooled radiofrequency ablation with corticosteroid injection in the management of knee pain from osteoarthritis. Reg Anesthesia Pain Med. 2018;43(1):1-08

${ }^{5}$ Rauck R, Deer T, Rosen S et al. Long-term follow-up of a novel implantable programmable infusion pump. Neuromodulation. 2013; 16(2):163-7

${ }^{6}$ Ma Xin-Long, Xing D, Ma Jian-Xiong et al. Balloon kyphoplasty versus percutaneous vertebroplasty in treating osteoporotic vertebral compression fractures: grading the evidence through a systematic review and meta-analysis. Eur Spine J. 2012;21:1844-59

${ }^{7}$ Genev I, Tobin M, Zaidi S et al. Spinal compression fracture management: A review of current treatment strategies and possible future avenues. Global Spine J. 2017;7(1):71-82

${ }^{8}$ Buchbinder R, Golmohammedi K, Johnston R et al. Percutaneous vertebroplasty for osteoporotic vertebral compression fracture. Cochrane Database Syst Rev. 2015;4:LCD0066349

${ }^{9}$ Manchikanti L, Kaye A, Knezevic N, et al. Responsible, safe, and effective prescription of opioids for chronic non-cancer pain: American Society of Interventional Pain Physicians (ASIPP Guidelines). Pain Physician. 2017;209:S3-S92

${ }^{10}$ Langley PC. Outcomes, registries and medical marijuana: Towards establishing dispensary monitoring and reporting standards. Inov Pharm. 2018;9(4):No.7

${ }^{11}$ Langley PC. Establishing outcomes credibility for medical marijuana dispensaries: Questions legislators should ask. Inov Pharm. 2019; 10(1): No. 1

12 Langley PC. Establishing credibility for medical marijuana: The proposed Prometheus Dispensary Registry for botanical cannabis. Inov Pharm. 2019;10(1):No. 2

${ }^{13}$ Romanelli R, Shah S, Ikeda L et al. Patient characteristics and healthcare utilization of a chronic pain population within an Integrated Healthcare System. Am J Manag Care. 2017;23(2):e50-e56

${ }^{14}$ Langley PC. Intelligent Design and Imaginary Worlds in Cost-Effectiveness Claims: An Overview of Commentaries in INNOVATIONS in Pharmacy from July 2016 to February 2017. Inov Pharm. 2017;8(1): No. 22

${ }^{15}$ Hurst H, Bolton J. Assessing the clinical significance of change scores recorded on subjective outcome measures. $J$ Manipulative Physiol Ther. 2004;27:26-35

${ }^{16}$ Rampakakis E, Ste-Marie P, Sampalis J et al. Real-life assessment of the validity of patient global impression of change in fibromyalgia. RMD Open. 2015;1:e000146

${ }^{17}$ Dworkin RH, Turk DC, Farrar JT et al. Core outcome measures for chronic pain clinical trials: IMMPACT recommendations. Pain, 2005;113:9-19. 
${ }^{18}$ Bartley EJ, Fillingim RB. Sex differences in pain: a brief review of clinical and experimental findings. Br J Anaesthesia. 2013;111(1):52-8

${ }^{19}$ Hollingshead NA, Ashburn-Nardo L, Stewart JC et al. The pain experience of Hispanic Americans: A critical literature review and conceptual model. J Pain. 2016; 17(5):513-28

${ }^{20}$ Campbell CM, Edwards RR. Ethnic differences in pain and pain management. Pain Manag. 2012;2(3):219-230

${ }^{21}$ Dionne CE, Von Korff M, Koepsell TD et al. Formal education and back pain: a review. J Epidemiol Community Health. 2001;55:45568

${ }^{22}$ Hagen KB, Tambs K, Bjerkedal T. What mediates the inverse association between education and occupational disability from back pain? A prospective cohort study from the Nord-Trøndelag Health Study in Norway. Soc Sci Med. 2006;3(5):1267-75

${ }^{23}$ Von Korff M, Ormel J, Keefe F et al. Grading the severity of chronic pain. Pain. 1992;50:133-49

${ }^{24}$ Von Korff M, Dunn K. Chronic pain reconsidered. Pain. 2008;138(2):267-76

${ }^{25}$ Bouhassira D, Attal N, Fermanian J et al. Development and validation of the neuropathic pain symptom inventory. Pain. 2004;108:248-57

${ }^{26}$ Portenoy R. Development and testing of a neuropathic pain screening questionnaire: ID Pain. Curr Med Res Opin. 2006;22(8):155565

${ }^{27}$ Freynhagen R, Baron R, Gockel U et al. painDETECT: a new screening questionnaire to identify neuropathic components in patients with back pain. Curr Med Res Opin. 2006;22(10):1911-20

${ }^{28}$ Bennett M, Smith B, Torrance N et al. The S-LANSS Score for identifying pain of predominantly neuropathic origin: Validation for use in clinical and postal research. J Pain. 2005;6(3):149-58

${ }^{29}$ Gudala K, Ghal B, Bansal D. Usefulness of four commonly used neuropathic pain screening questionnaires in patients with chronic low back pain: a cross-section study. Korean J Pain. 2017;30(1):51-8.

${ }^{30}$ Dawson J, Fitzpatrick R, Carr A et al. Questionnaire on the perceptions of patients about total hip replacement. Br J Bone Joint Surg. 1996;78-B(2):185-90

${ }^{31}$ Stewart WF. Validity of the Migraine Disability Assessment (MIDAS) Score in comparison to a diary-based measure in a population sample of migraine sufferers. Pain. 2000;88(1):41-52

${ }^{32}$ Aggarwal VR, Lunt M, Zakrzewska JM et al. Development and validation of the Manchester orofacial pain disability scale. Community Dent Oral Epidemiol. 2005;33:141-9

${ }^{33}$ Jordan A, Manniche C, Modsal C et al. The Copenhagen Functional Disability Scale: A study of reliability and validity. J Manipulative Physiol Ther. 1998;21(8):520-7

${ }^{34}$ Roach KE., Budiman-Mak E, Songsiridej N et al. Development of a shoulder pain and disability index. Arthritis Care Res. $1991 ; 4(4): 143-9$

${ }^{35}$ MacDermid JC. Outcome evaluation in patients with elbow pathology: issues in instrument development and evaluation. $J$ Hand Ther. 2001;14:105-114

${ }^{36}$ MacDermid, JC. The Patient-Rated Wrist/Hand Evaluation. School of Rehabilitation Science, McMaster University 2009 http://www.srsmcmaster.ca/Portals/20/pdf/research_resources/PRWHE.pdf 
${ }^{37}$ Roland M, Morris R. A study of the natural history of low back pain: Part 1. Development of a reliable and sensitive measure of disability in low back pain. Spine. 1983;8:141-4

${ }^{38}$ Roland M, Fairbank J. The Roland_Morris Disability Questionnaire and the Oswestry Disability Questionnaire. Spine. $2000 ; 25(24): 3115-24$

${ }^{39}$ Litwin MS, McNaughton-Collins M, Fowler FJ et al. The National Institutes of Health chronic prostatitis symptom index: development and validation of a new outcome measure. Chronic Prostatitis Collaborative Research Network. 1999. J Urol;162(2):369-75

${ }^{40}$ Greco, N. J., Anderson, A. F., Mann, et al. Responsiveness of the International Knee Documentation Committee subjective knee form in comparison to the Western Ontario and McMaster Universities Osteoarthritis Index, modified Cincinnati Knee Rating System, and Short Form 36 in patients with focal articular cartilage defects. Am J Sports Med. 2010; 38(5): 891-902.

${ }^{41}$ McCracken , LM, Hoskins J, Eccleston C. Concerns about medication and medication use in chronic pain. J Pain. 2006;7(10):726734. doi:10.1016/j.jpain.2006.02.014.

42 Elander J, Said O, Maratos FA, Dys A, Collins H, Schofield MB. Development and validation of a short-form Pain Medication Attitudes Questionnaire (PMAQ-14). Pain. 2017;158(3):400-407

${ }^{43}$ Adams L, Gatchel R, Robinson R et al. Development of a self-report screening instrument for assessing potential opioid medication misuse in chronic pain patients. J Pain Symptom Manag. 2004;27:440-59

${ }^{44}$ Butler SF, Fernandez K, Benoit C et al. Validation of the revised Screener and Opioid Assessment for Patients with Pain (SOAPP-R). J Pain. 2008;9(4):360-72

${ }^{45}$ Webster L, Webster R. Predicting aberrant behaviors in opioid-treated patients: preliminary validation pf the Opioid Risk Tool. Pain Med. 2005;6:432-42

${ }^{46}$ Jones T, Schmidt M, Moore T. Further validation of an opioid risk assessment tool: The Brief Risk Questionnaire. Ann Psychiatryt Ment Health. 2015;3(3):1032

47 Jones T, Lookatch S, Moore T. Validation of a new risk assessment tool: The Brief Risk Interview. J Opioid Manag. 2014;10:353-64

${ }^{48}$ Krupp LB, LaRocca NG, Muir-Bash J et al. The Fatigue Severity scale: applications to patients with multiple sclerosis and systemic lupus erythematosus. Arch Neurol. 1989;46:1121-3

${ }^{49}$ Schwartz JE, Jandorf L, Krupp LB. The measurement of fatigue: a new instrument. J Psychosom Res. 1993;37:753-62

${ }^{50}$ Kroenke K, Spitzer R, Williams JB. The PHQ-9: validity of a brief depression severity measure. J Gen Int Med. 2001;16:606-16

${ }^{51}$ Kroenke K, Spitzer R, Williams JBW et al. A brief measure for assessing generalized anxiety. Arch Int Med. 2006;166:1092-7

${ }^{52}$ Frank L, Kleinman L, Farup C et al. Psychometric validation of a constipation symptom assessment questionnaire. Scand J Gastroenterol. 1999;34:870-7

${ }^{53}$ Hays RD, Stewart AL. Sleep measures in A L Stewart and J E Ware (eds). Measuring functioning and well-being: The Medical Outcomes Study Approach (pp. 235-59). Durham, NC: Duke University Press, 1992

${ }^{54}$ Compton PA, Wu SM, Schieffer B et al. Introduction of a self-report version of the prescription drug use questionnaire and relationship to medication agreement non-compliance. J Pain Symptom Manag. 2008; 36(4):383-95

${ }^{55}$ Back SE, Payne R, Waldrop AE et al. Prescription opioid aberrant behavior: A pilot study of gender differences. Clin J Pain. 2009;25(6):477-81 
${ }^{56}$ Butler SF, Budman SH, Fernandez KC. Development and validation of the Current Opioid Misuse Measure. Pain. 2007;130(12):144-56

${ }^{57}$ Centers for Medicare and Medicaid services. MIPS Quality Measures. https://qpp.cms.gov/mips/quality-measures

${ }^{58}$ Centers for Medicare and Medicaid Services. MIPS Advancing Care Information. https://qpp.cms.gov/mips/advancing-careinformation

${ }^{59}$ Centers for Medicare and Medicaid Services. MIPS Improvement Activities https://www.cms.gov/Medicare/Quality-Payment-Program/Resource-Library/2018-Resources.html.)

${ }^{60}$ American Academy of Pain Management. http://www.painmed.org/practicemanagement/codingnews/get-ready-for-icd-10-cm$\underline{2018 /}$ 